\title{
Dimension of the Non-Differentiability Subset of the Cantor Function
}

\author{
Muquan Yan \\ Department of Mathematics, South China University of Technology, Guangzhou, China \\ Email: 851050338@qq.com
}

How to cite this paper: Yan, M.Q. (2020) Dimension of the Non-Differentiability Subset of the Cantor Function. Journal of $A p$ plied Mathematics and Physics, 8, 107-114. https://doi.org/10.4236/jamp.2020.81009

Received: October 30, 2019

Accepted: January 5, 2020

Published: January 8, 2020

Copyright (C) 2020 by author(s) and Scientific Research Publishing Inc. This work is licensed under the Creative Commons Attribution International License (CC BY 4.0).

http://creativecommons.org/licenses/by/4.0/

\begin{abstract}
The main purpose of this note is to estimate the size of the set $T_{\mu \lambda}$ of points, at which the Cantor function is not differentiable and we find that the Hausdorff dimension of $T_{\mu \lambda}$ is $[\log 2 / \log 3]^{2}$. Moreover, the Packing dimension of $T_{\mu \lambda}$ is $\log 2 / \log 3$. The $\log 2=\log _{e} 2$ is that if $a^{x}=N \quad(a>0$, and $a \neq 1$ ), then the number $x$ is called the logarithm of $N$ with a base, recorded as $x=\log _{a} N$, read as the logarithm of $N$ with a base, where a is called logarithm Base number, $N$ is called true number.
\end{abstract}

\section{Keywords}

Hausdor Dimension, Packing Dimension, Cantor Ternary Function, Cantor Ternary Set

\section{Introduction}

Let $T$ be the Cantor middle thirds set (Start with the closed interval $[0,1]$. Remove the open interval $\left(\frac{1}{3}, \frac{2}{3}\right)$ to obtain $\left[0, \frac{1}{3}\right] \cup\left[\frac{2}{3}, 1\right]$, i.e. two disjoint closed segments. Remove the middle thirds of those two segments, and you end up with four disjoint segments. After infinitely many steps, the result is called the Cantor set.) and $\theta$ be the Cantor ternary function (The function can be defined in the following way, given $x \in[0,1]$ consider its ternary expansion $a_{i}$, i.e. a choice of coefficients $a_{i} \in\{0,1,2\}$ such that

$$
x=\sum_{i=1}^{\infty} \frac{a_{i}}{3^{i}} .
$$

Define $n(x)$ to be $\infty$ if none of the coefficients $a_{i}$ takes the value 1 and the smallest integer $\mathrm{n}$ such that $a_{n}=1$ otherwise. 
Then

$$
f(x)=\sum_{i=1}^{n(x)-1} \frac{a_{i}}{2^{i+1}}+\frac{1}{2^{n(x)}} .
$$

Let $N^{*}$ be the set of non-differentiable points of Cantor function $\theta$.

Denote

$$
\theta_{-}=\liminf _{r \rightarrow 0^{-}} \frac{\theta(t+r)-\theta(t)}{r} .
$$

and

$$
\theta_{+}=\liminf _{r \rightarrow 0^{+}} \frac{\theta(t+r)-\theta(t)}{r} .
$$

See [1], we have

$$
\liminf _{r \rightarrow 0} \frac{\theta(t+r)-\theta(t)}{r}=\infty
$$

So,

$$
N^{*}=\left\{t \in T: \theta_{-}(t)<\infty \text { or } \theta_{+}(t)<\infty\right\} .
$$

Let $t=. t_{1} t_{2} \cdots$ be the ternary expansion of a point in $T$, which is not an endpoint of a complementary interval. Let $z(n)$ denote the position of the nth zero in this ternary expansion, and $t(n)$ denote the position of the nth two in the expansion of $t$.

For each $\mu \geq 0$ and $\lambda \geq 0$, define

$$
T_{\mu \lambda}=\left\{t \in T: \theta_{-}(t)=\mu \text { and } \theta_{+}(t)=\lambda\right\} .
$$

Firstly, several authors [2] [3] [4] have proved that the Hausdorff dimension of the set $N^{*}$ is $(\log 2 / \log 3)^{2}$ and the packing dimension of $N^{*}$ is $\log 2 / \log 3$. Let the Cantor $T$ in $R$ be defined by $T=\bigcup_{j=1}^{r} h_{j}(T)$ with a disjoint union, where the $h_{j}$ are similitude mappings with ratios $0<a_{j}<1$. Let $\mu$ be the selfsimilar Borel probability measure on $T$ corresponding to the probability vector $\left(p_{0}, p_{1}, \cdots, p_{r}\right)$. Let $S$ be the set of points at which the probability distribution function $F(x)$ of $\mu$ has no derivative, finite or infinite. On the one hand, for the case where $p_{j}>a_{j}$, Wen xia [5] has been studied the packing and box dimensions of $\mathrm{S}$ and give an approach to calculate the Hausdorff dimension of $S$. Kennen J. Falconer [6] is further noted that Hausdorff dimension of the set of points of non-differentiability of a self-affine "devil's staircase" function is the square of the dimension of the set of points of increase. On the other hand when $r=2$, for the case where $p_{1}<a_{1}$ or $p_{2}<a_{2}$. Yuanyuan yao, YunXin zhang and wenxia [1] Systematic has been given the Hausdorff and packing dimension of S. Secondly, Reza Mirzaire [7] find an upper bound for the Hausdorff dimension of the nondifferentiability set of a continuous convex function defined on a Riemannian manifold. As an application, he show that the boundary of a convex open subset of $R^{n}, n \geq 2$, has Hausdorff dimension at most $n-2$ and David 
Pavlica [8] characterize sets of non-differentiability points of convex functions on $R^{n}$. This completes (in $R^{n}$ ) the result by [9] which gives a characterization of the magnitude of these sets. Last, Eidswick [10] points out that the points of nondifferentiability of the Cantor ternary function are characterized in terms of the spacing of 0 's and 2's in ternary expansions, and have calculated that $T_{\mu \lambda}$ has the cardinality of the continuum.

Since about the dimension of the set $T_{\mu \lambda}$ of points, Eidswick didn't study it. So, our main job in this paper is to give its dimension, that is, the following theorem.

Theorem 1.1. $\operatorname{dim}_{\mathrm{H}} T_{\mu \lambda}=(\log 2 / \log 3)^{2}$, and $\operatorname{dim}_{\mathrm{P}} T_{\mu \lambda}=\log 2 / \log 3$.

\section{Proofs of Theorem}

First, we give a proposition, which simplifies the calculation of $\theta_{+}$and $\theta_{-}$.

Proposition 2.1. Let $t=._{1} t_{2} \cdots t_{n}$ be ternary expansion of $t$, and define

$$
\begin{gathered}
e_{n}=t_{1} t_{2} \cdots t_{z(n)-1} 2, \\
d_{n}=._{1} t_{2} \cdots t_{z(n)-1} 1 .
\end{gathered}
$$

Let

$$
Q_{n}:=\frac{\theta\left(e_{n}\right)-\theta(t)}{e_{n}-t} \text { and } P_{n}:=\frac{\theta\left(d_{n}\right)-\theta(t)}{d_{n}-t}
$$

we have

$$
\theta_{+}(t)=\liminf _{n \rightarrow \infty} Q_{n}
$$

and

$$
\theta_{-}(t)=\liminf _{n \rightarrow \infty} P_{n} \text {. }
$$

Proof. We just need to prove (2.3), since (2.4) is proved in a similar way.

If

$$
\liminf _{\delta \rightarrow 0} \frac{\theta(t+\delta)-\theta(t)}{\delta}=+\infty,
$$

then, the proposition is apparently true.

So, we suppose that

$$
\liminf _{\delta \rightarrow 0} \frac{\theta(t+\delta)-\theta(t)}{\delta}=c<+\infty,
$$

then, we can find $\left\{\delta_{n}\right\}_{n=1}^{+\infty}$ such that

$$
\lim _{n \rightarrow \infty} \frac{\theta\left(t+\delta_{n}\right)-\theta(t)}{\delta_{n}}=\liminf _{\delta \rightarrow 0} \frac{\theta(t+\delta)-\theta(t)}{\delta} .
$$

Note that $e_{n+1}+\frac{1}{3^{Z(n+1)}}=d_{n}$ and $\lim _{n \rightarrow+\infty} d_{n}=t$, we have

$$
\left(t, e_{1}+\frac{1}{3^{z(1)}}\right)=\bigcup_{n=1}^{+\infty}\left[d_{n}, e_{n}+\frac{1}{3^{z(n)}}\right) .
$$


Therefore, for any $k \geq 1$, there is an integer $n_{k}$, such that $t+\delta_{k} \in\left[d_{n_{k}}, e_{n_{k}}+\frac{1}{3^{z\left(n_{k}\right)}}\right)$.

By (2.5), for any $\varepsilon>0$, there is an integer $N>0$ such that: when $k>N$,

$$
\frac{\theta\left(t+\delta_{k}\right)-\theta(t)}{\delta_{k}}<c+\varepsilon .
$$

Denote $\eta_{k}=t+\delta_{k}-e_{n_{k}}$, then $0<\eta_{k}<3^{-z\left(n_{k}\right)}$, we have

$$
\theta\left(t+\delta_{k}\right)=\theta\left(e_{n_{k}}+\eta_{k}\right)=\theta\left(e_{n_{k}}\right)+\theta\left(\eta_{k}\right) .
$$

So,

$$
\frac{\theta\left(e_{n_{k}}\right)-\theta(t)}{e_{n_{k}}-t}=\frac{\theta\left(t+\delta_{k}\right)-\theta\left(\eta_{k}\right)-\theta(t)}{\delta_{k}-\eta_{k}},
$$

and since $\lim _{k \rightarrow+\infty} \frac{\theta\left(\eta_{k}\right)}{\eta_{k}}=+\infty$, it follows that

$$
\liminf _{k \rightarrow+\infty} \frac{\theta\left(e_{n_{k}}\right)-\theta(t)}{e_{n_{k}}-t} \leq \lim _{k \rightarrow+\infty} \frac{\theta\left(t+\delta_{k}\right)-\theta(t)}{\delta_{k}} .
$$

On the other hand, it is obvious that:

$$
\liminf _{k \rightarrow+\infty} \frac{\theta\left(e_{n_{k}}\right)-\theta(t)}{e_{n_{k}}-t} \geq \liminf _{\delta \rightarrow 0} \frac{\theta(t+\delta)-\theta(t)}{\delta},
$$

so we complete the proof of (2.3).

Besides, we need the following Lemma due to Billingsley:

Lemma 2.2. Let $b \geq 2$ be an integer and for $x \in[0,1]$, let $I_{n}(x)$ be the $n t h$ generation half-open $b$-adic interval of the form $\left[\frac{j-1}{b^{n}}, \frac{j}{b^{n}}\right]$ cintaining $x$, let $A \subset[0,1]$ be Borel and let $\mu$ be a finite Borel measure on $[0,1]$, suppose $\mu(A)>0$. If

$$
\alpha \leq \liminf _{n \rightarrow \infty} \frac{\log \mu\left(I_{n}(x)\right)}{\log \left|I_{n}(x)\right|} \leq \beta
$$

then

$$
\alpha \leq \operatorname{dim}_{\mathrm{H}}(A) \leq \beta \text {. }
$$

For the proof of this lemma, we refer readers to Section 1.4 of [11].

Corollary 2.3. For $S \subset N$, define

$$
A_{S}=\left\{x: \sum_{k \in S} x_{k} 3^{k}: x_{k} \in\{0,2\}\right\} .
$$

We have

$$
\operatorname{dim}_{\mathrm{H}}\left(A_{S}\right)=\lim _{N \rightarrow \infty} \frac{\#(S \cap\{1, \cdots, N\})}{N} \cdot \frac{\log 2}{\log 3} .
$$


Proof. To see this, let $\mu$ be the probability measure on $A_{S}$ that give equal measure to nth generation covering intervals. This measure makes the digits $\left\{x_{k}\right\}_{k \in S}$ in (2.6) independent identically distributed uniform random bits. For any $x \in A_{S}$,

$$
\frac{\log \mu\left(I_{n}(x)\right)}{\log \left|I_{n}(x)\right|}=\frac{\log 2^{-\#(S \cap\{1, \cdots, N\})}}{\log 3^{-n}}=\frac{\#(S \cap\{1, \cdots, N\})}{N} \cdot \frac{\log 2}{\log 3} .
$$

Thus the liminf of the left-hand side is the liminf of the right-hand side. By Lemma 2.2, this proves the corollary.

Last, we need to introduce a theorem and a formula:

Theorem 2.4. Let $t$ be a point of $T$ which is not an endpoint of a comple-mentary interval, let $z(n)$ denote the position of the nth zero in its ternary expansion, and let $\lambda=\liminf _{n \rightarrow \infty} \frac{3^{z}(n)}{2(n+1)}$. Then $\lambda \leq \theta_{+}(t) \leq 2 \lambda$. Furthermore, if $z(n+1)-z(n) \rightarrow \infty$, then, $\theta_{+}(t)=\lambda$.

Let, $Q_{n}=\frac{\theta\left(e_{n}\right)-\theta(t)}{e_{n}-t}$. Then,

$$
Q_{n}=\frac{3^{z(n)}}{2^{z(n+1)}} \frac{\sum_{1}^{\infty} 2^{-(z(n+k)-z(n+1))}}{1+2 \sum_{1}^{\infty} 3^{-(z(n+k) z(n))}}
$$

The proof of theorem 2.4 and formula (2.8), we see [10].

Proof of Theorem. First, let's construct a subset of $T_{\mu \lambda}$.

Let $\mu_{0}=\frac{\log \mu}{\log 2}, \lambda_{0}=\frac{\log \lambda}{\log 2}$. We can find increasing sequences of positive integers $\left(a_{n}\right)$ and $\left(b_{n}\right)$ such that

$$
a_{2 n} \xi-b_{2 n} \rightarrow \lambda_{0},
$$

and

$$
a_{2 n+1} \xi-b_{2 n+1} \rightarrow \mu_{0},
$$

when $n$ is large enough, where $\xi=\frac{\log 3}{\log 2}$. At the same time, we may request that $a_{n}<b_{n}, \quad a_{n+1}-b_{n} \geq 4 n$ and $b_{n} \geq 3 n$ hold for all $n \geq 1$. Moreover, denote $M=\left[\frac{a_{2 n+1}-b_{2 n}}{2 n}\right]$ and $M^{*}=\left[\frac{a_{2 n+2}-b_{2 n+1}}{2 n}\right]$, we insert these points $\left(b_{2 n, i}\right)_{i=1}^{M}$ and $\left(b_{2 n+1, j}\right)_{j=1}^{M^{*}}$ in to $\left(b_{2 n}, a_{2 n+1}\right)$ and $\left(b_{2 n+1}, a_{2 n+2}\right)$, respectively, such that

$$
b_{2 n, i+1}-b_{2 n, i}=2 n \text {, }
$$

and

$$
b_{2 n+1, j+1}-b_{2 n+1, j}=2 n,
$$

when $n$ is large enough, for $i=1,2, \cdots, M$ and $j=1,2, \cdots, M^{*}$; and let $b_{2 n}=b_{2 n, 0}, b_{2 n+1,0}=b_{2 n+1}$. We will show the selection of $\left\{a_{n}\right\}$ and $\left\{b_{n}\right\}$ later. Define $t_{m}=0$, if 
$m \in\left\{a_{2 n}, b_{2 n}, b_{2 n, 1}, \cdots, b_{2 n, M}, b_{2 n+1,1}+1, b_{2 n+1,2}+1, \cdots, b_{2 n+1, M^{*}}+1\right\}$, or

$a_{2 n+1}<m<b_{2 n+1}$.

Define $t_{m}=0$, if

$m \in\left\{a_{2 n+1}, b_{2 n+1}, b_{2 n+1,1}, b_{2 n+1,2}, \cdots, b_{2 n+1, M^{*}}, b_{2 n, 1}+1, b_{2 n, 2}+1, \cdots, b_{2 n, M}+1\right\}$, or

$a_{2 n}<m<b_{2 n}$.

And restrict $t_{m} \in\{0,2\}$ (That is, $t_{m}$ is free to take a value of 0 or 2 for all other values of $\mathrm{m}$ ).

And we claim that $t=\sum_{m=1}^{\infty} \frac{t_{m}}{3^{m}} \in T_{\mu \lambda}$. We'll give a simple proof after the selection of $\left(a_{n}\right)_{n=1}^{+\infty}$ and $\left(b_{n}\right)_{n=1}^{+\infty}$, let $\Omega$ be the set of all such $t$.

For the selection of $\left(a_{n}\right)_{n=1}^{+\infty},\left(b_{n}\right)_{n=1}^{+\infty}$, first of all, take $a_{0}=b_{0}=1$. Assume that $a_{2 n}$ and $b_{2 n}$ is taken, we choose $a_{2 n+1}$, such that

(a) $a_{2 n+1} \geq 8 n+b_{2 n}$;

(b) $\left[\xi a_{2 n+1}\right]-\left[\mu_{0}\right]>a_{2 n+1}$ (note that $\xi>1$ );

(c) $\left|\left\{\xi a_{2 n+1}\right\}-\left\{\mu_{0}\right\}\right|<\frac{1}{n}$ (note that $(\xi n)$ is uniformly distributed modulo $1)$.

where $[x]$ represents the integer part that represents taking $x$, and $\{y\}$ represents the fractional portion of the $y$. And take $b_{2 n+1}=\left[\xi a_{2 n+1}\right]-\left[\mu_{0}\right]$. We choose $a_{2 n+2}$ such that

(d) $a_{2 n+2} \geq 4(2 n+1)+b_{2 n+1}$;

(e) $\left[\xi a_{2 n+2}\right]-\left[\lambda_{0}\right]>a_{2 n+2}$;

(f) $\left|\left\{\xi a_{2 n+2}\right\}-\left\{\lambda_{0}\right\}\right|<\frac{1}{n+1}$.

and take $b_{2 n+2}=\left[\xi a_{2 n+2}\right]-\left[\lambda_{0}\right]$.

These $\left(a_{n}\right)_{n=1}^{+\infty}$ and $\left(b_{n}\right)_{n=1}^{+\infty}$ meet the desired conditions.

From $a_{2 n} \xi-b_{2 n} \rightarrow \lambda_{0}$ and $a_{2 n+1} \xi-b_{2 n+1} \rightarrow \mu_{0}$, we can get that

$$
\frac{3^{a_{2 n}}}{2^{b_{2 n}}} \rightarrow \lambda \text { and } \frac{3^{a_{2 n+1}}}{2^{b_{2 n+1}}} \rightarrow \mu
$$

To see that $t=\sum_{m=1}^{\infty} \frac{t_{m}}{3^{m}} \in T_{\mu \lambda}$, by the Theorem 2.4 and formula (2.8), we only need to show that $b_{2 n}$ and $a_{2 n}$ are adjacent positions of zeros, whose spacing is largest; and $b_{2 n+1}$ and $a_{2 n+1}$ are adjacent positions of twos, whose spacing is largest.

Since $a_{2 n} \rightarrow \frac{b_{2 n}}{\xi}+\frac{\lambda}{\xi}$, for any $\epsilon>0$, when $n$ is large enough, we have

$$
\left|a_{2 n}-\frac{b_{2 n}}{\xi}-\frac{\lambda}{\xi}\right|<\epsilon
$$

then, when $n$ is large enough

$$
b_{2 n}-a_{2 n}>b_{2 n}-\frac{b_{2 n}}{\xi}-\frac{\lambda_{0}}{\xi}-\varepsilon=\left(1-\frac{1}{\xi}\right) b_{2 n}-\frac{\lambda_{0}}{\xi}-\varepsilon \approx 0.36 b_{2 n}>2 n .
$$

Then, according to our above-mentioned construction of $t=\sum_{m=1}^{\infty} \frac{t_{m}}{3^{m}}$, other 
spacings of zeros are at most $2 n$, so $b_{2 n}-a_{2 n}$ is the largest spacing of zeros. Similarly, we have $b_{2 n+1}-a_{2 n+1}>2 n$, and $b_{2 n+1}-a_{2 n+1}$ is the largest spacing of twos.

Denote

$$
\begin{gathered}
A_{n}=\left(\left\{k \in N: b_{2 n} \leq k \leq a_{2 n+1}\right\} \bigcup\left\{k \in N: b_{2 n+1} \leq k \leq a_{2 n+1}\right\}\right), \\
B_{n}=\left(\left(\bigcup_{i=1}^{M}\left\{b_{2 n, i}, b_{2 n, i}+1\right\}\right) \cup\left(\bigcup_{j=1}^{M^{*}}\left\{b_{2 n+1, j}, b_{2 n+1, j}+1\right\}\right)\right) .
\end{gathered}
$$

Let

$$
B:=\bigcup_{n=1}^{+\infty}\left(A_{n} \backslash B_{n}\right)
$$

in fact, the set $\mathrm{B}$ is all positions that $t_{m}$ can freely select 0 or 2 in the above construction. Using Corollary 2.3, we have

$$
\operatorname{dim}_{\mathrm{p}} \Omega=\operatorname{dim}_{\mathrm{B}} \Omega=\limsup _{N \rightarrow \infty} \frac{\#\{k \in B, k \leq N\}}{N} \cdot \frac{\log 2}{\log 3},
$$

and

$$
\operatorname{dim}_{\mathrm{H}} \Omega=\liminf _{N \rightarrow \infty} \frac{\#\{k \in B, k \leq N\}}{N} \cdot \frac{\log 2}{\log 3} .
$$

And we calculate these as follows: It's not hard to see that, when $N=a_{2 n+1}$, we can get the superior limit:

$$
\underset{N \rightarrow \infty}{\limsup } \frac{\#\{k \in B, k \leq N\}}{N} \cdot \frac{\log 2}{\log 3}=\lim _{n \rightarrow \infty} \frac{a_{2 n+1}-b_{2 n}-2 M}{a_{2 n+1}} \cdot \frac{\log 2}{\log 3}=\frac{\log 2}{\log 3}
$$

where $\lim _{n \rightarrow \infty} \frac{b_{2 n}}{a_{2 n+1}}=0, \lim _{n \rightarrow \infty} \frac{M}{a_{2 n+1}}=0$.

On the other hand, when $N=b_{n}$, we can get the inferior limit:

$$
\operatorname{dim}_{\mathrm{H}} \Omega=\liminf _{N \rightarrow \infty} \frac{\#\{k \in B, k \leq N\}}{N} \cdot \frac{\log 2}{\log 3}=\lim _{n \rightarrow \infty} \frac{\#\left\{k \in B, k \leq b_{n}\right\}}{n} \cdot \frac{\log 2}{\log 3},
$$

and by calculating

$$
\lim _{n \rightarrow \infty} \frac{\#\left\{k \in B, k \leq b_{2 n+1}\right\}}{b_{2 n+1}} \cdot \frac{\log 2}{\log 3} \geq \lim _{n \rightarrow \infty} \frac{a_{2 n+1}-b_{2 n}-2 M}{b_{2 n+1}} \cdot \frac{\log 2}{\log 3}=(\log 2 / \log 3)^{2}
$$

where $\lim _{n \rightarrow \infty} \frac{a_{2 n+1}}{b_{2 n+1}}=\frac{\log 2}{\log 3}, \lim _{n \rightarrow \infty} \frac{b_{2 n}}{b_{2 n+1}}=0$ and $\lim _{n \rightarrow \infty} \frac{M}{b_{2 n+1}}=0$;

$$
\lim _{n \rightarrow \infty} \frac{\#\left\{k \in B, k \leq b_{2 n+2}\right\}}{b_{2 n+2}} \cdot \frac{\log 2}{\log 3} \geq \lim _{n \rightarrow \infty} \frac{a_{2 n+2}-b_{2 n+1}-2 M^{*}}{b_{2 n+2}} \cdot \frac{\log 2}{\log 3}=(\log 2 / \log 3)^{2}
$$

where $\lim _{n \rightarrow \infty} \frac{a_{2 n+2}}{b_{2 n+2}}=\frac{\log 2}{\log 3}, \lim _{n \rightarrow \infty} \frac{b_{2 n+1}}{b_{2 n+2}}=0, \lim _{n \rightarrow \infty} \frac{M^{*}}{b_{2 n+2}}=0$.

In Section 1, we already know $\operatorname{dim}_{\mathrm{p}} N^{*}=\log 2 / \log 3$, and $\operatorname{dim}_{\mathrm{H}} N^{*}=(\log 2 / \log 3)^{2}$.

Since $\Omega \subset T_{\mu \lambda} \subset N^{*}$, we have $\operatorname{dim}_{\mathrm{H}} \Omega=\operatorname{dim}_{\mathrm{H}} T_{\mu \lambda}=(\log 2 / \log 3)^{2}$ and 
$\operatorname{dim}_{\mathrm{P}} \Omega=\operatorname{dim}_{\mathrm{P}} T_{\mu \lambda}=\log 2 / \log 3$.

\section{Conclusion}

Finally, through the above proof, we solved dimension of the set $T_{\mu \lambda}$ of points that is Eidswick didn't study it. In other words, we finally get that $\operatorname{dim}_{\mathrm{H}} T_{\mu \lambda}=(\log 2 / \log 3)^{2}$, and $\operatorname{dim}_{\mathrm{P}} T_{\mu \lambda}=\log 2 / \log 3$.

\section{Conflicts of Interest}

The author declares no conflicts of interest regarding the publication of this paper.

\section{References}

[1] Yao, Y.Y., Zhang, Y.X. and Li, W.X. (2009) Dimension of Non-Differentiability Points of Cantor Functions. Studia Mathematica, 195, 113-125.

https://doi.org/10.4064/sm195-2-2

[2] Bandt, C. and Graf, S. (1992) Self-Similar Sets. VII. A Characterization of Self-Similar Fractals with Positive Hausdorff Measure. Proceedings of the American Mathematical Society, 114, 995-1001. https://doi.org/10.1090/S0002-9939-1992-1100644-3

[3] Li, W.X. (2004) Non-Differentiability Points of Cantor Functions. East China Normal University, Shanghai.

https://onlinelibrary.wiley.com/doi/pdf/10.1002/mana.200410470

[4] Darst, R. (1993) The Hausdorff Dimension of the Non-Differentiability Set of the Cantor Function Is $[\ln 2 / \ln 3]^{2}$. Proceedings of the American Mathematical Society, 119, 105 108. https://doi.org/10.2307/2159830

[5] Li, W.X. (2007) Non-Dierentiability Points of Cantor Function. Mathematische Nachrichten, 280, 140-151. https://doi.org/10.1002/mana.200410470

[6] Falconer, K.J. (2002) One-Sided Multifractal Analysis and Points of Non-Differentiability of Devils Staircases. University of St Andrews, St Andrews.

[7] Mirzaie, R. (2017) Hausdorff Dimension of the Nondifferentiability Set of a Convex Function. Filomat, 31, 5827-5831. https://doi.org/10.2298/FIL1718827M

[8] Pavlica, D. (2004) On the Points of Non-Dierentiability of Convex Functions. Commentationes Mathematicae Universitatis Carolinae, 4, 727-734.

[9] Zajíček, L. (1991) Praha Smallness of Sets of Nondifferentiability of Convex Functions in Non-Separable Banach Spaces. Commentationes Mathematicae Universitatis Carolinae, 41, 288-296.

[10] Eidswick, J.A. (1974) A Characterization of the Nondifferentiability Set of the Cantor Function. Proceedings of the American Mathematical Society, 42, 214-217. https://doi.org/10.1090/S0002-9939-1974-0327992-8

[11] Bishop, C.J. and Peres, Y. (2017) Fractals in Probability and Analysis. Cambridge University Press, Cambridge. 